\title{
Cloxacillin-induced acute vanishing bile duct syndrome: a case study and literature review
}

\author{
Kyrillos Faragalla ${ }^{1}$, Helena $\mathrm{Lau}^{2}$, Hanlin Wang ${ }^{3}$, and Jimin $\mathrm{Liu}^{4}$ \\ ${ }^{1}$ University of Saskatchewan \\ ${ }^{2}$ Halton Healthcare Services \\ ${ }^{3}$ University of California Los Angeles David Geffen School of Medicine \\ ${ }^{4}$ McMaster University Faculty of Health Sciences
}

March 3, 2022

\begin{abstract}
Ductopenia is often regarded as a chronic process where [?]50\% of portal tracts lack bile ducts, which is also known as vanishing bile duct syndrome (VBDS). One etiology is drug-induced liver injury. Cloxacillin, an anti-staphylococcal penicillin, typically causes "bland" cholestasis. We present the first case of cloxacillin-induced acute ductopenia or VBDS and a review of published cloxacillin-induced liver injuries. A 66-year-old woman with no prior liver disease, but known penicillin allergy, was treated for post-carotid angioplasty staphylococcal infection with 6 weeks of cloxacillin. She presented with a two-week history of weakness and jaundice. Laboratory work-up showed elevated liver enzymes, hyperbilirubinemia, and eosinophilia. She required ICU transfer for hypotension and was started empirically on prednisone. Liver biopsy revealed severe centrilobular cholestasis, mild necroinflammation, and ductopenia with epithelial injury, but no ductular reaction. Two-months later, she was discharged on hydrocortisone and ursodiol with persistently elevated alkaline phosphatase and bilirubin. She was considered for liver transplantation but died of liver failure four months later. Four additional articles were found with histopathologic descriptions of cloxacillin-related liver injury. These included portal inflammation, cholestasis and mild necroinflammation. Clinical features were reported in two cases; both had mild symptoms with cholestatic liver enzymes and hyperbilirubinemia. Both patients recovered completely within 10-60 days. Cloxacillin-induced cholestasis can be secondary to acute ductopenia, which can result in worse clinical outcomes than previously described "bland" cholestasis. Liver biopsy is recommended to identify cases with acute VBDS.
\end{abstract}

Title : Cloxacillin-induced acute vanishing bile duct syndrome: a case study and literature review

Running Title : A case of cloxacillin-induced VBDS

Authors : Kyrillos Faragalla ${ }^{1}$, Helena Lau ${ }^{2}$, Hanlin L. Wang ${ }^{3}$, Jimin $\mathrm{Liu}^{4,5}$

1. Internal Medicine, University of Saskatchewan, Saskatoon, ON, Canada S7N 5A2. kyrillos.faragalla@medportal.ca

2. Gastroenterology, Halton Healthcare, 3001 Hospital Gate, Oakville, ON, Canada L6M 0L8. hlau@haltonhealthcare.com

3. Pathology and Laboratory Medicine, David Geffen School of Medicine, University of California Los Angeles, 10833 Le Conte Ave, Los Angeles, CA, United States 90095. hanlinwang@mednet.ucla.edu

4. Laboratory Medicine, Halton Healthcare, 3001 Hospital Gate, Oakville, ON, Canada L6M 0L8. liuj107@mcmaster.ca

5. Pathology and Molecular Medicine, Faculty of Health Sciences, McMaster University, 1280 Main St W, Hamilton, ON, Canada L8S 4L8. liuj107@mcmaster.ca 
Keywords : cloxacillin, DILI, ductopenia, cholestasis, VBDS

Ethics Statements

None of the authors have a conflict of interest. No funding was required for this project. Verbal consent for publication was obtained from a family member as the patient was deceased.

What's New?

Cloxacillin is known to cause acute or "bland" liver cholestasis. This case study highlights that it can also cause vanishing bile duct syndrome or ductopenia, which can be more persistent with a worse prognosis.

Abstract

Ductopenia is often regarded as a chronic process where [?]50\% of portal tracts lack bile ducts, which is also known as vanishing bile duct syndrome (VBDS). One etiology is drug-induced liver injury. Cloxacillin, an anti-staphylococcal penicillin, typically causes "bland" cholestasis. We present the first case of cloxacillininduced acute ductopenia or VBDS and a review of published cloxacillin-induced liver injuries.

A 66-year-old woman with no prior liver disease, but known penicillin allergy, was treated for post-carotid angioplasty staphylococcal infection with 6 weeks of cloxacillin. She presented with a two-week history of weakness and jaundice. Laboratory work-up showed elevated liver enzymes, hyperbilirubinemia, and eosinophilia. She required ICU transfer for hypotension and was started empirically on prednisone. Liver biopsy revealed severe centrilobular cholestasis, mild necroinflammation, and ductopenia with epithelial injury, but no ductular reaction. Two-months later, she was discharged on hydrocortisone and ursodiol with persistently elevated alkaline phosphatase and bilirubin. She was considered for liver transplantation but died of liver failure four months later.

Four additional articles were found with histopathologic descriptions of cloxacillin-related liver injury. These included portal inflammation, cholestasis and mild necroinflammation. Clinical features were reported in two cases; both had mild symptoms with cholestatic liver enzymes and hyperbilirubinemia. Both patients recovered completely within 10-60 days.

Cloxacillin-induced cholestasis can be secondary to acute ductopenia, which can result in worse clinical outcomes than previously described "bland" cholestasis. Liver biopsy is recommended to identify cases with acute VBDS.

\section{Introduction}

Ductopenia is defined by loss of [?]50\% intrahepatic bile ducts. For an accurate diagnosis on a liver biopsy, at least 11 portal tracts need to be evaluated ${ }^{1-2}$. Ductopenia is associated with a wide variety of liver pathologies including congenital and genetic diseases such as biliary atresia and cystic fibrosis, autoimmune diseases such as primary biliary cholangitis (PBC) and primary sclerosing cholangitis (PSC), infections such as CMV and HIV, neoplasms such as Hodgkin lymphoma, ischemic insults, and injuries induced by a large array of drugs or toxins ${ }^{3}$. However, some cases have no identifiable etiology, a phenomenon previously recognized as idiopathic adulthood ductopenia but now better known as vanishing bile duct syndrome (VBDS) $)^{3-4}$. These conditions typically present with chronic cholestasis, although isolated transaminitis has been reported ${ }^{5-6}$.

Drug-induced liver injury (DILI) can be divided into hepatitic, cholestatic or mixed hepatitic and cholestatic patterns clinically. Microscopically, DILI varies from necroinflammatory injury, cholestatic injury, steatosis or steatohepatitis, fibrosis with cirrhosis, vascular lesions, neoplasia, to other rare patterns ${ }^{7}$. Drug-induced cholestatic liver injury also takes several forms. The mildest form is acute cholestasis or bland cholestasis, which shows intrahepatic cholestasis with minimal inflammation. When intrahepatic cholestasis is accompanied by necroinflammation and duct injury, it is cholestatic hepatitis ${ }^{8}$. Drug-induced cholestatic liver injury may also be chronic, and can be further divided into PSC-like, PBC-like, or VBDS pattern ${ }^{8-9}$. The VBDS pattern was initially recognized with chlorpromazine, but many other drugs were later found to be associated with ductopenia ${ }^{9-11}$. Ductopenia diagnosed on liver biopsy has been reported as early as three weeks after 
the onset of symptoms ${ }^{12}$. Some sources also distinguish between acute and chronic drug-induced VBDS, the latter characterized histologically by the presence of ductular reaction and copper accumulation ${ }^{7}$. In certain cases, jaundice and abnormal liver function tests may eventually resolve, and successful treatments have been reported ${ }^{13-15}$. However, VBDS may also progress to biliary cirrhosis and require liver transplantation $(\mathrm{LT})^{12,16-18}$.

Cloxacillin, oxacillin, and flucloxacillin belong to a group of penicillinase-resistant isoxazolyl penicillins which are often used for methicillin-sensitive Staphylococcus species,Pneumococcus, and betahemolyticStreptococci $i^{19}$. Flucloxacillin is a well-known cause of drug-induced cholestasis and particularly ductopenia $^{12,20-23}$. In contrast, oxacillin and cloxacillin have only been reported in cases of acute intrahepatic cholestasis, with bile accumulation in hepatocytes or canaliculi without duct loss, or cholestatic hepatitis with associated inflammation ${ }^{24-26}$. We present the first case of cloxacillin-induced acute ductopenia and review the clinicopathologic features of reported cases of cloxacillin-related liver injury.

\section{Case Report}

This was a 66-year-old female who was initially admitted to a different hospital for balloon angioplasty of a partially thrombosed internal carotid artery. Following the procedure, she developedStaphylococcus hominis bacteremia and was prescribed six weeks of cloxacillin. Three weeks after completing her antibiotic course, she presented to our hospital with a two weeks history of generalized weakness and jaundice. Her past medical history additionally included gastroesophageal reflux disease, for which she was on pantoprazole twice daily, and hypopituitarism for which she was on levothyroxine and hydrocortisone. She had no personal or family history of liver disease, alcohol overconsumption, or IV drug use. She had allergies to codeine and penicillin. Jaundice and scleral icterus were noted on physical examination.

On admission, her liver enzymes were markedly elevated with a mixed pattern: aspartate aminotransferase (AST) $822 \mathrm{U} / \mathrm{L}$ (5-34 U/L), alanine aminotransferase (ALT) $877 \mathrm{U} / \mathrm{L}$ (5-55U/L), and alkaline phosphatase (ALP) $4279 \mathrm{U} / \mathrm{L}$ (40-150 U/L). Her total bilirubin level was also high, $752 \mathrm{umol} / \mathrm{L}$ (3.4-20.5 umol/L) and predominantly conjugated, $588.3 \mathrm{umol} / \mathrm{L}(1.6-8.6 \mathrm{umol} / \mathrm{L})$. A blood routine test showed leukocytosis, 12.6 x $10^{9} / \mathrm{L}\left(4.0-11.0 \times 10^{9} / \mathrm{L}\right)$ with eosinophilia, $1.2 \times 10^{9} / \mathrm{L}\left(0.0-0.1 \times 10^{9} / \mathrm{L}\right)$, as well as microcytic anemia: hemoglobin $95 \mathrm{~g} / \mathrm{L}(115-155 \mathrm{~g} / \mathrm{L})$ and mean corpuscular volume $78.5 \mathrm{fL}$ (82-97 fL). Workup for hemolysis as well as other causes of acute transaminitis was unremarkable. During her admission, she displayed signs of hepatic encephalopathy and she became hypotensive necessitating ICU admission. It was unclear whether this was related to acute liver failure or adrenal insufficiency. Abdominal ultrasound and CT scan showed no hepatosplenomegaly or ascites and were otherwise unremarkable. She was started empirically on oral prednisone (40mg daily) for presumed hepatotoxicity and underwent a liver needle biopsy on post-admission day 15.

The biopsy specimen was adequate for histopathologic evaluation, containing 19 portal tracts. In the lobules, there was severe zone 3 and focal zone 2 cholestasis in hepatocytes and dilated canaliculi (Figure 1A), patchy hepatocyte feathering degeneration, and scattered lymphocytes and neutrophils. The portal tracts revealed mild or no inflammation. However, 13 portal tracts had no interlobular bile ducts nor ductular reaction (Figure 1B), which was further confirmed by CK7 immunostain (Figure 1B inset). The remaining bile ducts showed features of epithelial degeneration characterized by cytoplasmic eosinophilia, nuclear pleomorphism, uneven spacing of nuclei, pyknotic nuclei, and cytoplasmic vacuoles (Figures 1C and 1D). There was no cholate stasis nor interface hepatitis. Copper stain (Rhodanine) was negative. Given the clinical history of recent cloxacillin use, the biopsy findings were compatible with acute cholestatic liver injury with bile duct injury and loss secondary to cloxacillin. The differential also included ischemic cholangiopathy secondary to her hypotensive episode, however the lack of ischemic changes at zone 3 hepatocytes makes this less likely.

After spending more than two months in hospital, the patient was discharged. In addition to her admission medications, she was discharged on ursodiol (250mg three times per day for cholestasis), lactulose (30ml three times per day for hepatic encephalopathy), N-acetylcysteine (600mg daily), and Slow-K (1200mg daily). Her hydrocortisone was tapered down from stress dose to total $15 \mathrm{mg}$ daily because her adrenal insufficiency was 
stable. During follow-up, her liver enzymes, particularly ALP level, remained elevated and an outpatient MRCP revealed beaded appearance of the common bile duct and the left intrahepatic bile duct. She was assessed by a liver transplantation center for potential LT. Unfortunately, about two months after discharge, this patient had a fall and developed multiple fractures. She passed away after two more months hospital stay. The final cause of death was drug-induced liver failure. No autopsy was performed.

\section{Discussion}

Our case report illustrates a new type of cloxacillin-induced liver injury, acute vanishing bile duct syndrome. Strengths of this study include the collaboration between the clinicians and pathologists in managing this patient by having liver biopsy to determine the specific pattern of her cholestasis and liver failure, as opposed to undifferentiated DILI. The patient was also followed closely as an outpatient by her medical providers, including LT team, which provided information about the persistence of her disease after discharge. One limitation of this study is the fact that the patient reported a penicillin allergy, which may have made her more susceptible to a cloxacillin reaction than non-allergic patients, though reported penicillin allergies are often unreliable ${ }^{27}$. The other limitation is that patient unfortunately had a fall and developed multiple traumatic fractures, which likely contributed and accelerated deterioration of her liver disease and her passing away before getting the LT.

Using Google Scholar, we found four additional cases of cloxacillin-induced livery injury requiring a liver biopsy. One retrospective study examined a series of drug rechallenge cases, from the Global Safety Database, where patients who had possible DILI were given the same drug while monitoring liver enzymes ${ }^{27}$. One of the 88 confirmed positive rechallenge cases was caused by cloxacillin-related injury, and liver biopsy showed a portal lymphocytic infiltrate with some eosinophils ${ }^{28}$. A similar retrospective study examined 77 possible or probable DILI cases secondary to penicillinase-resistant penicillins reported to the Swedish Adverse Drug Reactions Advisory Committee ${ }^{29}$. One case related to cloxacillin underwent liver biopsy which showed cholestasis with moderate inflammation including some eosinophilic infiltrate ${ }^{29}$. No specific demographic or clinical information was reported for either of those two cases.

There have been only two case reports of cloxacillin-induced liver injury that included both clinical and histopathologic information ${ }^{25-26}$. Both patients were female, aged 69 and 77 years, and presented with jaundice and pruritis. Additionally, the first patient had hepatomegaly and the second had weakness and maculopapular rash. They both had a cholestatic liver injury pattern, but their ALP (1126 and 394), AST (18 and 313) and total bilirubin (255 and 221) levels were lower than those seen in our case. Eosinophilia was only noted in the second patient. Both liver biopsies showed moderate to severe intrahepatic cholestasis, which was canalicular in the first case and hepatocellular in the second. Both also had up to moderate portal inflammation, and mild lobular necroinflammation. Additionally, the first case showed occasional hepatocyte variation and multinucleation, Kupffer cell hyperplasia with lipofuscin and ceroid pigmentation, and minimal steatosis. Normal bile ducts were seen in the first case and no abnormalities were documented in the second. Prednisone was only used for the second patient, but both had normal liver enzymes and recovered clinically within 60 and 10 days, respectively.

Altogether, lobular or portal inflammation was described in all four reported cases of cloxacillin-induced liver injury, whereas eosinophils were documented in three cases. Three patients had cholestasis with moderate to severe or prominent cholestasis in canalicular or hepatocellular patterns in two cases. Bile ducts were reported as normal in one case and assumed to be normal in the other three. Both cases that were followed clinically had a full recovery.

The prognosis of drug-induced VBDS has been variable in general. In a case series of 8 patients with druginduced VBDS followed with sequential liver biopsies, three recovered clinically, three recovered biochemically and clinically, and one developed biliary cirrhosis ${ }^{30}$. In a 10-year retrospective study, from 2004 to 2014 , by the US DILI network, 26 of $363(7 \%)$ patients that underwent a liver biopsy had bile duct loss (14 lost >50\% bile ducts, while 12 lost $25-50 \%$ bile ducts) ${ }^{31}$. Compared to patients without bile duct loss, patients with bile duct loss had a higher risk of developing chronic liver disease, persistent cholestasis after six months 
(94\% vs $47 \%$ ), and death within two years of follow up (27\% vs $9 \%)$.

Treatment for drug-induced VBDS is largely similar to that for other types of drug-induced cholestasis, including immediate withdrawal of the culprit drug and preventing re-exposure, as well as ursodexycholic acid to protect against toxic bile salts ${ }^{15,32-33}$. Steroids and other immunosuppressants may be used when there is an indication of allergic reaction such as eosinophilia, lymphadenopathy, rash or syndromes like Stevens-Johnson syndrome ${ }^{33}$. Plasmapheresis has been used successfully in a case of loxprofen-induced ductopenia in an adolescent female unresponsive to initial treatment measures ${ }^{14}$. In cases that progress to biliary cirrhosis, supportive management and LT would be indicated ${ }^{18}$.

Our case shows that cloxacillin-induced cholestasis can be secondary to acute ductopenia, which is associated with more severe clinical presentations and worse outcome than the previously described bland cholestasis. Liver biopsy is therefore important to helping identify patients with VBDS early in the disease process. A lower threshold for liver transplantation assessment is recommended for those cases.

\section{Acknowledgement}

No funding sources were sought for this project.

Conflict of Interest

None of the authors have a conflict of interest.

\section{References}

1. Ldwig J. Idiopathic adulthood ductopenia: an update. Mayo Clin Proc. 1998;73:285-91.

2. Rockey DC, Caldwell SH, Goodman Z, Nelson RC, Smith AD. Liver biopsy. Hepatol. 2009;49:1017-44.

3. Reau NS, Jensen DM. Vanishing bile duct syndrome. Clin Liver Dis. 2008;12:203-17.

4. Ludwig J, Wiesner RH, LaRusso NF. Idiopathic adulthood ductopenia. A cause of chronic cholestatic liver disease and biliary cirrhosis. J Hepatol. 1988;7:193-9.

5. Bilal M, Kazemi A, Babich M. Idiopathic Adulthood Ductopenia: 'It Is Out There'. Case Rep Gastroenterol. 2016;10:99-102.

6. Zhang XC, Wang D, Li X, Hu YL, Wang C. Idiopathic adulthood ductopenia with elevated transaminase only: A case report. World J Clin Cases. 2020;8:6524-8.

7. Burt A, Ferrell L, Hubscher S. MacSween's Pathology of the Liver. 7th ed. Philadelphia: Elsevier; 2018.

8. Kleiner DE. The Pathology of Drug-Induced Liver Injury. Semin Liver Dis. 2009;29:364-72.

9. Padda MS, Sanchez M, Akhtar AJ, Boyer JL. Drug-induced cholestasis. Hepatology. 2011;53:1377-87.

10. Moradpour D, Altorfer J, Flury R, Greminger P, Meyenberger C, Jost R, Schmid M. Chlorpromazineinduced vanishing bile duct syndrome leading to biliary cirrhosis. Hepatology. 1994;20:1437-41.

11. Desmet VJ. Vanishing bile duct syndrome in drug-induced liver disease. J Hepatol. 1997;26 Suppl $1: 31-5$.

12. Davies MH, Harrison RF, Elias E, Hübscher SG. Antibiotic-associated acute vanishing bile duct syndrome: a pattern associated with severe, prolonged, intrahepatic cholestasis. J Hepatol. 1994;20:112-6.

13. Schumaker AL, Okulicz JFMeropenem-induced vanishing bile duct syndrome. Pharmacotherapy. 2010;30:953.

14. Kawasaki Y, Matsubara K, Hashimoto K, Tanigawa K, Kage M, Iwata A, et al. Nonsteroidal antiinflammatory drug-induced vanishing bile duct syndrome treated with plasmapheresis. J Pediatr Gastroenterol Nutr. 2013;57: e30-1.

15. Cho HJ, Jwa HJ, Kim KS, Gang DY, Kim JY. Urosodeoxycholic Acid Therapy in a Child with Trimethoprim-Sulfamethoxazole-induced Vanishing Bile Duct Syndrome. Pediatr Gastroenterol Hepatol Nutr. 2013;16:273-8.

16. Ishii M, Miyazaki Y, Yamamoto T, Miura M, Ueno Y, Takahashi T, et al. A case of drug-induced ductopenia resulting in fatal biliary cirrhosis. Liver. 1993;13:227-31. 
17. Douglass KM, Willner IR, Glenn DJ, Jones RM. Idiopathic Adulthood Ductopenia Causing Cirrhosis. ACG Case Rep J. 2020;7:e00349.

18. Hashim A, Barnabas A, Miquel R, Agarwal K. Successful liver transplantation for drug-induced vanishing bile duct syndrome. BMJ Case Rep. 2020;13:e233052.

19. Sutherland R, Croydon EA, Rolinson GN. Flucloxacillin, a new isoxazolyl penicillin, compared with oxacillin, cloxacillin, and dicloxacillin. Br Med J. 1970;4:455-60.

20. Devereaux BM, Crawford DH, Purcell P, Powell LW, Roeser HP. Flucloxacillin associated cholestatic hepatitis. An Australian and Swedish epidemic?. Eur J Clin Pharmacol. 1995;49:81-5.

21. Andrews E, Daly AK. Flucloxacillin-induced liver injury. Toxicology. 2008;254:158-63.

22. Eckstein RP, Dowsett JF, Lunzer MR. Flucloxacillin induced liver disease: histopathological findings at biopsy and autopsy. Pathology. 1993;25:223-8.

23. Nghona N, Sonderup MW, Spearman CWN. Jaundice and an itch following a fall. Contin Med Educ. 2013;31:270-1.

24. Pas AT, Quinn EL. Cholestatic hepatitis following the administration of sodium oxacillin. JAMA. 1965;191:674-5.

25. Enat R, Pollack S, Ben-Arieh Y, Livni E, Barzilai D. Cholestatic jaundice caused by cloxacillin: macrophage inhibition factor test in preventing rechallenge with hepatotoxic drugs. $\mathrm{Br}$ Med $\mathrm{J}$. 1980;280:982-3.

26. Goland S, Malnick SD, Gratz R, Feldberg E, Geltner D, Sthoeger ZM. Severe cholestatic hepatitis following cloxacillin treatment. Postgrad Med J. 1998;74:59-60.

27. Stone CA, Trubiano J, Coleman DT, Rukasin CRF, Phillips EJ. The challenge of de-labeling penicillin allergy. Allergy. 2020;74:273-288.

28. Papay JI, Clines D, Rafi R, Yuen N, Britt SD, Walsh JS, et al. Drug-induced liver injury following positive drug rechallenge. Regul Toxicol Pharmacol. 2009;54:84-90.

29. Olsson R, Wiholm BE, Sand C, Zettergren L, Hultcrantz R, Myrhed M. Liver damage from flucloxacillin, cloxacillin and dicloxacillin. J Hepatol. 1992;15:154-61.

30. Degott C, Feldmann G, Larrey D, Durand-Schneider AM, Grange D, Machayekhi JP, et al. Druginduced prolonged cholestasis in adults: a histological semiquantitative study demonstrating progressive ductopenia. Hepatology. 1992;15:244-51.

31. Bonkovsky HL, Kleiner ED, Gu J, Odin JA, Russo MW, Navarro VM, et al. Clinical presentations and outcomes of bile duct loss caused by drugs and herbal and dietary supplements. Hepatology. 2017;65:1267-77.

32. Perez MJ, Briz O. Bile-acid-induced cell injury and protection. World J Gastroenterol. 2009;15:167789.

33. Bessone F, Hernandez N, Tanno M, Roma MG. Drug-Induced Vanishing Bile Duct Syndrome: From Pathogenesis to Diagnosis and Therapeutics. Semin Liver Dis. 2021;41:331-48.

Figure Legends

Figure 1 : Figure 1 A) Zone 3 cholestasis, H\&E 40x; B) Ductal injury, H\&E 40x; Portal tract in C) H\&E 40x and D) CK7 stain 40x.

\section{Hosted file}

Figure 1_Faragalla et al.docx available at https://authorea.com/users/463364/articles/558463cloxacillin-induced-acute-vanishing-bile-duct-syndrome-a-case-study-and-literaturereview 